\title{
Central cortical cleanup and zonular deficiency
}

\author{
Ahmad M Mansour ${ }^{1,2}$ \\ Rafic S Antonios' \\ lqbal Ike K Ahmed ${ }^{3}$ \\ 'Department of Ophthalmology, \\ American University of Beirut, \\ Beirut, Lebanon; ${ }^{2}$ Department \\ of Ophthalmology, Rafic Hariri \\ University Hospital, Beirut, Lebanon; \\ ${ }^{3}$ Department of Ophthalmology, \\ University of Toronto, Toronto, ON, \\ Canada
}

Correspondence: lqbal Ike K Ahmed Department of Ophthalmology, University of Toronto, 27 King's College Circle, Toronto, ON M5S, Canada Email ikeahmed@mac.com
This article was published in the following Dove Press journal:

Clinical Ophthalmology

4 October 2016

Number of times this article has been viewed

Background: Complete removal of the cortex has been advocated to prevent posterior capsular opacification but carries the risk of zonular dehiscence, hence there is a need for a safe maximal cortical cleanup technique in eyes with severe diffuse zonulopathy in subjects above age 90.

Methods: We used bimanual central cortical cleaning by elevating central fibers and aspirating them toward the periphery. Peripheral cortical fibers were removed passively only when they became loose due to copious irrigation. A one-piece foldable implant was inserted without a capsular tension ring. Postoperative corticosteroid drops were used.

Results: This technique was safely performed in a dozen eyes with severe pseudo-exfoliation or brunescent cataract with weak zonules. Posterior capsular rupture, iritis, vitreous loss, and lens subluxation were not observed. Moderate capsular phimosis occurred but with maintained central vision.

Conclusion: The dogma of "complete cortical cleanup" in severe zonulopathy needs to be revisited in favor of a clear visual axis with maximal preservation of the damaged zonules. This technique is ideal in patients above age 90 where posterior capsular opacification and late dislocation of intraocular lens-capsule bag complex are unlikely to occur until several years postoperatively.

Keywords: brunescent cataract, cortex aspiration, phacoemulsification, pseudo-exfoliation, weak zonules

\section{Introduction}

The circumferential suspensory ligament of the lens, or zonular apparatus, has been well-known to anatomists since 1755 when Johann Zinn identified its fibrillar nature. ${ }^{1-4}$ Thereafter, the "zonule of Zinn" or ciliary zonule has been the subject of further investigation with the application of transmission and scanning electron microscopy, as well as three-dimensional ultrabiomicroscopy. This elaborate system of fibers suspends the lens from the nonpigmented ciliary epithelium and comprises approximately 140 zonule bundles. FBN1, a large glycoprotein enriched in force-bearing tissues, is a prominent constituent of the zonule in various species allowing extraordinary distension capacity of the zonular fibers..$^{2-4}$ However, with advanced age, the number of zonules decreases with increase in fragility of the zonular fibrils. Moreover, certain diseases are associated with zonular weakness, the most common one being pseudoexfoliation followed by ultra-brunescent cataracts, ocular trauma, surgical trauma (vitrectomy, repeated intravitreal injection), high myopia, retinitis pigmentosa, Marfan syndrome, and uveitis. Schlötzer-Schrehardt and Naumann ${ }^{5}$ tried to clarify and localize the cause of zonular weakness in pseudo-exfoliation syndrome by using scanning and transmission electron microscopy. The pseudo-exfoliation material infiltrated the zonular bundles passing alongside the ciliary processes leading to zonular rupture; also at their attachment to the anterior lens capsule, the zonular lamella were focally lifted and subsequently ruptured by pseudo-exfoliation masses erupting through the capsular surface. Pseudo-exfoliation is present in approximately half of subjects above 
80 years of age, being occult in one quarter of the subjects (occurring at the lens periphery). ${ }^{6}$ Weak zonules complicate every step of the cataract procedure and challenge surgeons to diagnose and manage intraoperative zonulopathy. Once it has been determined that a patient has zonular weakness, the surgeon must decide whether to proceed with surgery while being extra cautious, or whether to use adjunctive devices to help support the capsule. There are basically two permanent devices to choose from: a capsular tension ring (CTR) or capsule support hooks. In addition, in severe zonular laxity, temporary capsule retractors can help to stabilize the capsular bag in the axial direction, permit rotation of the nucleus, and do not impede aspiration of the cortex, yet with some complications. Standard extensive cortex aspiration can increase zonular damage and hence the need to adopt safer techniques.

\section{Technique}

Good phacoemulsification outcomes depend on obtaining a clear central optical visual pathway. In eyes with deficient zonules, excess cortex aspiration can lead to zonular dialysis, difficulty in implant insertion, and late implant subluxation. ${ }^{7}$ Appropriate techniques of the bimanual irrigation-aspiration surgical step of phacoemulsification in these difficult eyes need to address ways to minimize centripetal traction exerted on the zonular fibers. We can partially address these concerns by generous hydrodissection and copious irrigation of the cortex, ${ }^{8}$ as well as tangential stripping of the aspirated cortex. ${ }^{9}$ The authors have also been using a bimanual centrifugal central cortical cleaning technique in eyes with more severe zonular dehiscence and miosis (Figure 1). Following generous irrigation, the cortex is gently engaged centrally under low suction and elevated in a centrifugal direction for a distance of $3 \mathrm{~mm}$ (Figure 2) and aspirated in tiny pieces without attempt at pulling at the posterior capsule. This "nibbling" maneuver (Video S1) is repeated until the central $5 \mathrm{~mm}$ optical axis is free of cortical fibers. Postoperative use of a corticosteroid has helped to control anterior chamber reaction to the residual peripheral cortical fibers. We have been using this technique in a dozen patients over the past 5 years with moderate capsular phimosis, but no posterior capsular opacification, and no implant subluxation.

The institutional review board of Rafic Hariri University Hospital gave ethical approval for this methodology study and report. A written informed consent was obtained from all participants for inclusion in this paper.

\section{Case report}

This 92-year-old lady had advanced coronary artery disease and visual loss to finger counting bilaterally from ultrabrunescent cataract (Figure 2) and pseudo-exfoliation. Under pure (no sedation) topical anesthesia, the right eye underwent attempted phacoemulsification. There was excessive wrinkling and mobility of the anterior capsule. Extracapsular cataract extraction was done, and the capsule was spontaneously expressed. An anterior chamber implant was placed. Under topical anesthesia also, the left eye underwent phacoemulsification of the very dense nucleus. The cortex was aspirated from the center as described in the technique. A CTR was not placed as its insertion would traumatize the loose zonules. One day postoperatively the cornea was clear, and uncorrected visual acuity was 20/25. The implant was well centered. At the 1-month follow-up, moderate capsular phimosis (Figure 3) was observed with preservation of the central visual axis and absence of implant-donesis. This persisted at the last follow-up 6 months postoperatively.
A

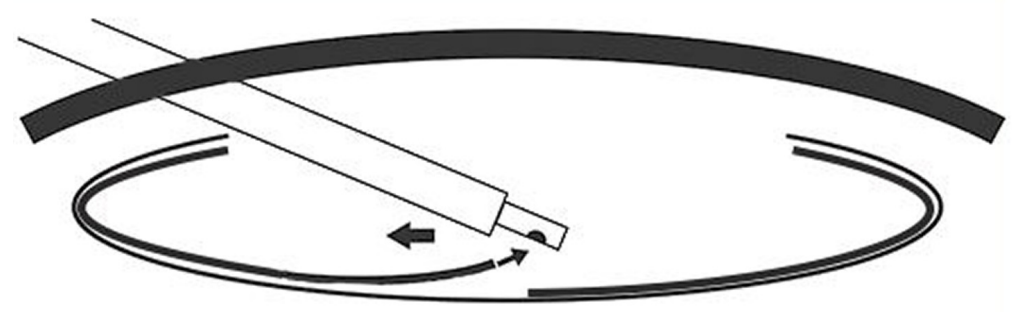

B

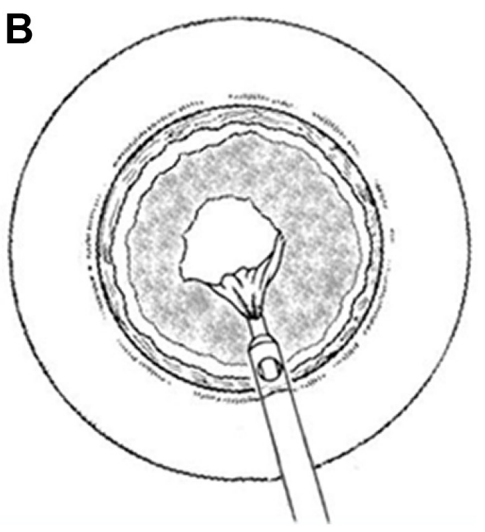

Figure I Schema of the technique of central cortical clean-up in eyes with severe zonular dehiscence and miosis. Notes: (A) Cross-section; (B) front view. The central fibers are engaged and aspirated toward the periphery. This is repeated until the central visual axis is clear. 

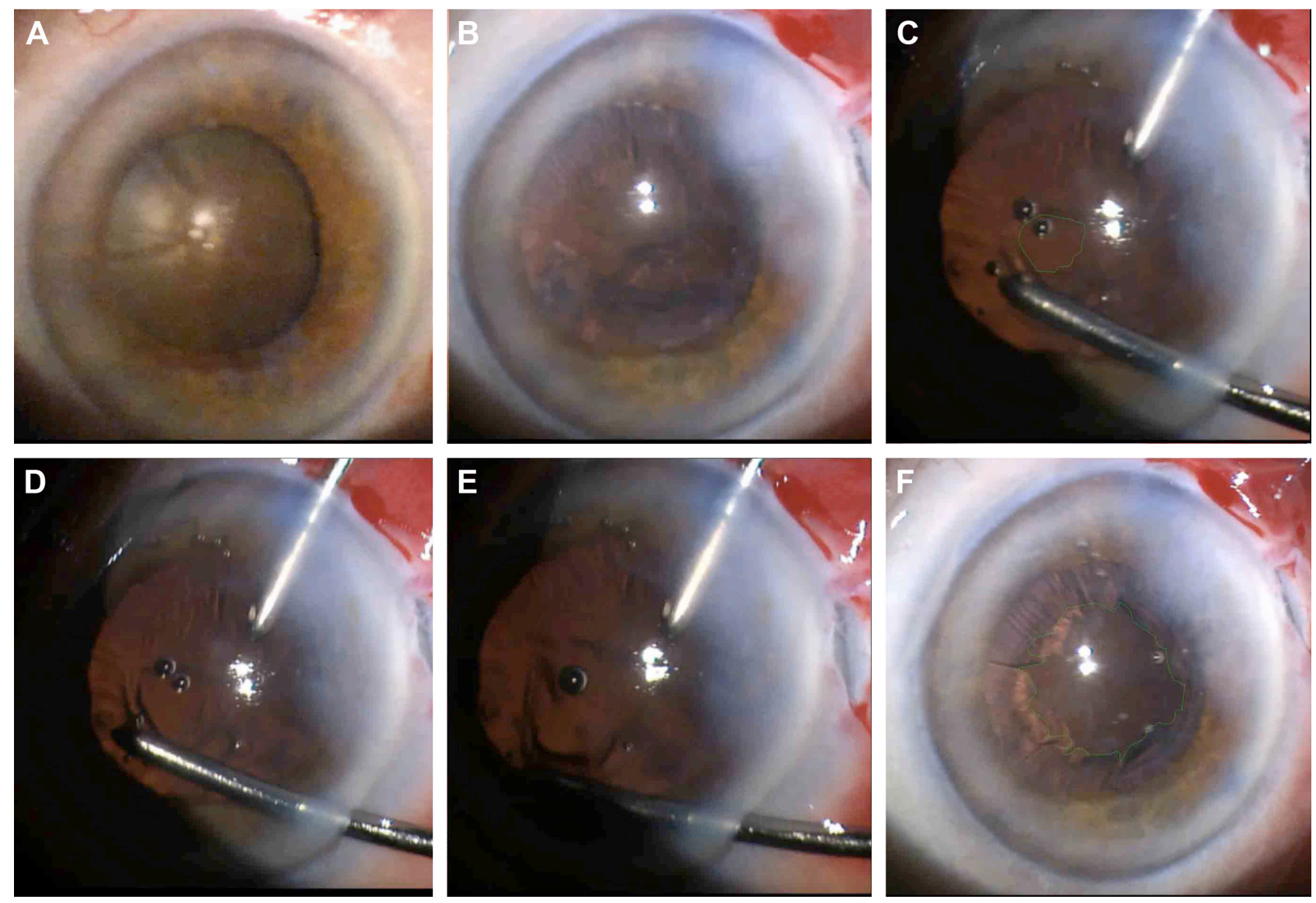

Figure 2 Ultra-brunescent cataract managed with phacoemulsification and central cortex aspiration.

Notes: (A) Ultra-brunescent cataract with pseudo-exfoliation and severe zonular weakness in the left eye, prior to fellow eye surgery. (B) Cortex fibers aspirated from the center toward the periphery. The cortex layer is intact. (C) Removal of central $2 \mathrm{~mm}$ of cortex with appearance of posterior capsule. (D) The cortex fibers are pulled centrifugally at around the 8 o'clock position from the center and aspirated under low settings, enlarging the cortex-free zone. (E) The aspiration is made at the edge at the 7 o'clock position with gentle centrifugal pull and the cortex is pulled and aspirated all around the central clear zone ("nibbling" technique). (F) Only the peripheral cortex remains. This is removed by copious irrigation attempting to loosen the cortex allowing aspiration of the loose fibers.

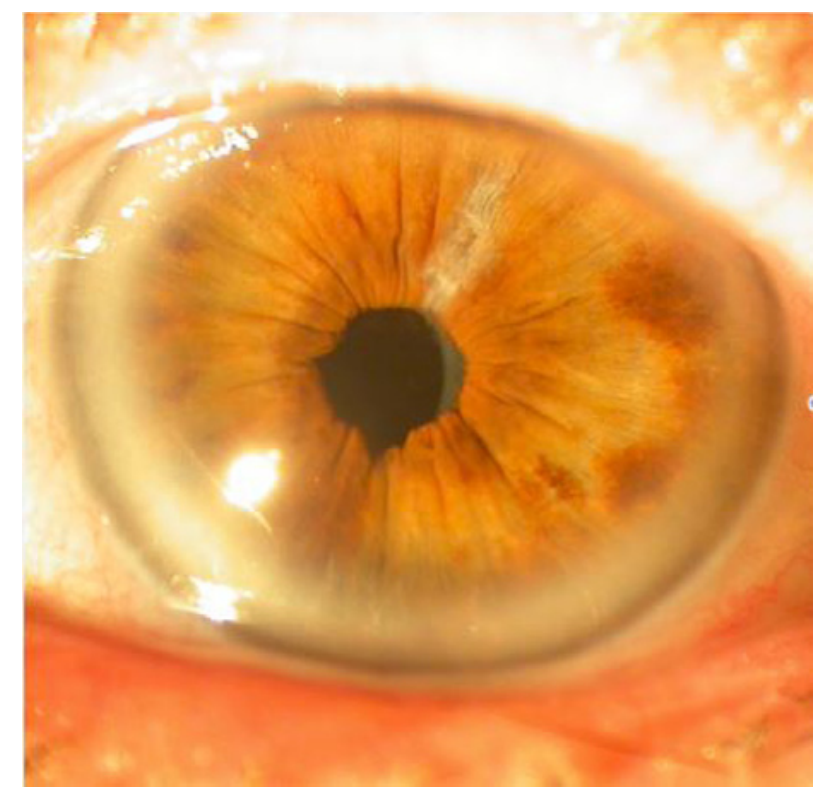

Figure 3 Capsular phimosis is present at the 2-month follow-up with uncorrected visual acuity of $20 / 25$.

\section{Discussion}

Alternative procedures in cataract surgery in eyes with severe zonular weakness include extracapsular cataract extraction, intracapsular cataract extraction, or phacoemulsification with placement of the implant in the sulcus (with or without lens optic capture), iris fixation or in-the-bag lens insertion along with placement of a CTR. ${ }^{10-13}$ Insertion of a CTR before cortex aspiration helps to counteract the traction on the zonules by the standard centripetal cortex aspiration technique and also decreases late posterior capsular opacification. ${ }^{10} \mathrm{CTR}$ reduces intraoperatively redundant capsule folds, forward trampolining of the posterior capsule, and inward collapsing of the capsular fornices toward the aspirating instrument tip. However, the CTR does not have an element for scleral fixation and is therefore used in dialysis up to 3-4 clock hours or with mild generalized zonular deficiency. ${ }^{13}$ Insertion of a CTR puts some zonules under stress and the weight of the CTR may be an additional 
cause for the subsequent dislocation of the implant-lens bag complex. ${ }^{14}$ However in severe diffuse zonulopathy, CTR could add to the risk of further subluxation of the complex intraocular lens-CTR ${ }^{11}$ Endocapsular support devices which permit scleral-suture fixation, including the modified CTR, the capsular tension segment, and the capsule stabilization hook implantation, have become increasingly important in the management of compromised zonules during cataract surgery. ${ }^{12,13}$ In the present 92-year-old patient, both eyes had an extremely severe zonulopathy and placement of CTR or regular cortex aspiration would end up in further compromise of the zonular apparatus. It is likely that fast development of posterior capsular opacification would not be a concern. Also, in-the-bag dislocation usually happens more than 5 years after surgery. Still, central cortical cleaning carries the risk of posterior capsular rupture if not done in a meticulous manner with low aspiration mode (especially with thin capsules in very advanced cataracts and with presence of capsular folds due to loss of zonular stretch).

Once again, we reiterate the crucial role of hydrodissection and copious irrigation of the cortex in any technique of cortical removal in eyes with weak zonules. ${ }^{15}$ Copious hydrodissection by creating a diffuse and complete posterior fluid wave with accompanying lifting or elevation of the nucleus is advocated in eyes with weak zonules for truly adequate cortical cleanup. ${ }^{8,15}$ This decreases the incidence of capsular rupture and maintains zonular integrity. As shown experimentally, hydrodissection remains the best available, practical, immediately implementable, and inexpensive means to help remove cortical fibers and decrease posterior capsular opacification. ${ }^{8,15}$

Thorough cortical aspiration has been advocated as an essential step to minimize postoperative anterior chamber inflammation and prevent posterior capsular opacification. ${ }^{8}$ Complete removal of the cortex is crucial in pediatric, juvenile, and young adult cataract surgery. However, in elderly subjects in their 90s with severe zonular disease, we advise against obsessive meticulous removal of stubborn peripheral fibers and use of gentle central cortical cleanup. The current technique avoids centripetal traction exerted on the zonular fibers. The benefit of maintaining zonular stability and leaving part of stubborn peripheral cortex outweighs the risk of postoperative inflammation that can be controlled by a topical corticosteroid. Central cleaning of the cortex can be used in miotic pupils when the cortex is not easily aspirated from the lens equator. The risk of posterior capsular opacification is present but can later be treated with a small central laser capsulotomy. The current technique needs further study with a large sample size and a long-term follow-up.

\section{Disclosure}

The study received no financial support. The authors report no conflicts of interest in this work.

\section{References}

1. Rohen JW. Scanning electron microscopic studies of the zonular apparatus in human and monkey eyes. Invest Ophthalmol Vis Sci. 1979;18(2):133-144.

2. Canals M, Costa-Vila J, Potau JM, Merindano MD, Ruano D. Scanning electron microscopy of the human zonule of the lens (Zonula ciliaris). Acta Anat (Basel). 1996;157(4):309-314.

3. Curtis R. The suspensory apparatus of the canine lens. J Anat. 1983; 136(Pt 1):69-83.

4. Shi Y, Tu Y, De Maria A, Mecham RP, Bassnett S. Development, composition, and structural arrangements of the ciliary zonule of the mouse. Invest Ophthalmol Vis Sci. 2013;54(4):2504-2515.

5. Schlötzer-Schrehardt U, Naumann GO. A histopathologic study of zonular instability in pseudoexfoliation syndrome. Am J Ophthalmol. 1994; 118(6):730-743.

6. Lumme P, Laatijkainen L. Exfoliation syndrome and cataract extraction. Am J Ophthalmol. 1993;116(1):51-55.

7. Agarwal T, Jhanji V, Gopalakrishnan K, Khokhar SK, Dada T, Panda A. Hybrid bimanual technique for aspiration of residual cortex. Acta Ophthalmol. 2012;90(3):e236-e2367.

8. Peng Q, Apple DJ, Visessook N, et al. Surgical prevention of posterior capsule opacification. Part 2: Enhancement of cortical cleanup by focusing on hydrodissection. J Cataract Refract Surg. 2000;26(2): 188-197.

9. Nakano CT, Motta AF, Hida WT, et al. Hurricane cortical aspiration technique: one-step continuous circular aspiration maneuver. $J$ Cataract Refract Surg. 2014;40(4):514-516.

10. D’Eliseo D, Pastena B, Longanesi L, Grisanti F, Negrini V. Prevention of posterior capsule opacification using capsular tension ring for zonular defects in cataract surgery. Eur J Ophthalmol. 2003;13(2):151-154.

11. Ahmed II, Chen SH, Kranemann C, Wong DT. Surgical repositioning of dislocated capsular tension rings. Ophthalmology. 2005;112(10): $1725-1733$.

12. Hasanee K, Ahmed II. Capsular tension rings: update on endocapsular support devices. Ophthalmol Clin North Am. 2006;19(4):507-519.

13. Asano Y, Yaguchi S, Nishimura E, Soda M, Kozawa T. Modified capsule expander implantation to reposition and fixate the capsular bag in eyes with subluxated cataractous lenses and phacodonesis: intermediate-term results. J Cataract Refract Surg. 2015;41(3):598-606.

14. Hayashi K, Hirata A, Hayashi H. Possible predisposing factors for inthe-bag and out-of-the-bag intraocular lens dislocation and outcomes of intraocular lens exchange surgery. Ophthalmology. 2007;114(5): 969-975.

15. Apple DJ, Solomon KD, Tetz MR, et al. Posterior capsule opacification. Surv Ophthalmol. 1992;37(2):73-116. 


\section{Supplementary material}

Video SI Case report of ultra-brunescent cataract managed with phacoemulsification and then central cortex aspiration for maximal preservation of the weak zonules.

Clinical Ophthalmology

\section{Publish your work in this journal}

Clinical Ophthalmology is an international, peer-reviewed journal covering all subspecialties within ophthalmology. Key topics include: Optometry; Visual science; Pharmacology and drug therapy in eye diseases; Basic Sciences; Primary and Secondary eye care; Patient Safety and Quality of Care Improvements. This journal is indexed on

PubMed Central and CAS, and is the official journal of The Society of Clinical Ophthalmology (SCO). The manuscript management system is completely online and includes a very quick and fair peer-review system, which is all easy to use. Visit http://www.dovepress.com/ testimonials.php to read real quotes from published authors. 